\title{
Intelligent Trajectory Tracking Control of Robot-Assisted Surgery
}

\author{
Mohammad Reza Avazpour, Farzin Piltan, Hooton Ghiasi, Mohammad Hadi \\ Mazloom and Amirzubir Sahamijoo \\ Intelligent System and Robotic Lab, Iranian Institute of Advance Science and Technology \\ (IRAN SSP), Shiraz/Iran \\ piltan_f@iranssp.com,WWW.IRANSSP.COM/english
}

\begin{abstract}
Robotic surgery, computer-assisted surgery, and robotically-assisted surgery are terms for technological developments that use robotic systems to aid in surgical procedures. Robotically-assisted surgery was developed to overcome the limitations of minimallyinvasive surgery and to enhance the capabilities of surgeons performing open surgery. In the case of robotically-assisted minimally-invasive surgery, instead of directly moving the instruments, the surgeon uses one of two methods to control the instruments; either a direct telemanipulator or through computer control. A telemanipulator is a remote manipulator that allows the surgeon to perform the normal movements associated with the surgery whilst the robotic arms carry out those movements using end-effectors and manipulators to perform the actual surgery on the patient. In computer-controlled systems the surgeon uses a computer to control the robotic arms and its end-effectors, though these systems can also still use telemanipulators for their input. One advantage of using the computerised method is that the surgeon does not have to be present, but can be anywhere in the world, leading to the possibility for remote surgery. The multi degrees of freedom actuator is an important joint, which has attracted worldwide developing interests for its medical, industry and aerospace applications. This paper addresses the problem of trajectory tracking of three dimensions joint in the presence of model uncertainties and external disturbances. An adaptive fuzzy sliding mode controller (AFLSMC) is proposed to steer a three dimension joint along a desired trajectory precisely. First, the dynamics model of a three dimension joint is formulated and the trajectory tracking problem is described. Second, a sliding mode controller (SMC) is designed to track a time-varying trajectory. The fuzzy logic system (FLS) is employed to approximate the uncertain model of the three dimension joint, with the tracking error and its derivatives and the commanded trajectory and its derivatives as FLS inputs and the approximation of the uncertain model as FLS output. And a fuzzy logic system is also adopted to attenuate the chattering results from the SMC. The control gains are tuned synchronously with the sliding surface according to fuzzy rules, with switching sliding surface as fuzzy logic inputs and control gains as fuzzy logic outputs. The stability and convergence of the closed-loop controller is proven using the Lyapunov stability theorem. Finally, the effectiveness and robustness of the proposed controller are demonstrated via simulation results. Contrasting simulation results indicate that the AFLSMC attenuates the chattering effectively and has better performance against the SMC.
\end{abstract}

Keywords: Telemanipulator, assisted surgery, robotically-assisted surgery, sliding mode control, fuzzy rules, approximation, adaptive method, multi degrees of freedom joints. 


\section{Introduction}

The use of robots in medical applications has increased considerably in the last decade. Today, there are robots being used in complex surgeries such as those of the brain, eye, heart, and hip. By one survey, 2285 medical robots were estimated to be in use at the end of 2002, and that number is expected to rise to over 8000 medical robots by 2006. It is also estimated that medical robots may, in the end, have the largest market value among all types of robots.

Complex surgeries have complex requirements, such as high precision, reliability over multiple and long procedures, ease of use for physicians and other personnel, and a demonstrated advantage, to the patient, of using a robot. Furthermore, all new technologies in the medical area have to undergo strict regulatory clearance procedures, which may include clinical trials, as outlined by various government regulatory agencies. In the U.S., the Food and Drug Administration (FDA) has jurisdiction over medical devices. As more and more devices get through the regulatory procedures, there will be more and more robots in the medical world. Before one can consider the usage of robots in medical applications, it is important to understand that medical applications have unique requirements, different from general, or "traditional," robot design. Some of the design issues and associated advantages are described below.

1. High Precision: Modern robots are demonstrated to be highly precise. The precision range depends on the robot and the application, of course, but it is generally accepted that for a given application, a robot can be designed to meet or exceed the precision requirements of the application. A typical industrial robot has repeatability specifications measured in tenths of a millimeter. A representative ratio of motion in robotic assisted surgery is that a $1 \mathrm{~cm}$ movement of a doctor's hand translates to a $0.1 \mathrm{~cm}$ movement of the robotic tool.

2. Heavy Payloads: Modern robots can carry heavy payloads over large workspaces, at high speeds, with high precision. Industrial robots are available with payload capacity of a few ounces to over $1000 \mathrm{lb}$.

3. Workspace: Medical robot workspace requirements tend to be significantly larger than industrial needs because of patient related factors, such as uncertainty in patient location during the procedure and safety requirements. There is an obvious overriding need to avoid any hazard to the patient, physician, and other medical personnel; this drives an exclusionary zone around the patient, doctor, and other equipment that may be attached to the patient. Thanks to the advances driven by industrial applications, the workspace of most available robots is significant and can be utilized for medical applications.

4. High Speed: Most new robots have been designed and optimized for industrial automation, enabling them to move at high speeds with high precision. The majority of medical applications do not require robots to move at high speeds as these robots are working on a patient. Reassurance, comfort, and safety dictate the robot's speed in medical applications.

5. Reliability: Industrial robots are designed to work round the clock without stopping; their medical counterparts work only a few hours a day. The nature of medical applications is that most of the time is taken up by other parts of the surgery, such as operating room preparation, patient preparation, and postoperative procedures. The robots actually perform surgeries for only a limited time, around $10 \%$ of surgery time. The resulting reliability numbers for medical work are excellent, leading to very limited downtime. 
6. Tedium: Most of the medical applications where robots are sought involve repetitive tasks over a very long period of time. Some surgeries last for many hours, during which the operators are required to repeat tasks hundreds or thousands of times. Obviously, robots do not have any problems with tedium.

7. High Quality: Robotic assisted surgery can help a wide variety of doctors perform complex surgeries with the same high quality previously achieved only by some accomplished surgeons. Additionally, most medical procedures cannot tolerate any degradation in quality due to trembling or unsteadiness of hands. Robotic systems in the operating room can compensate for imperfections in the user due to age, fatigue, or other factors, without degrading the quality of care administered to the patient.

8. Computer Control: Robotic surgery is able to capitalize on available diagnostic data to calculate an optimized approach to treatment. Most modern systems use fusion of multiple imaging modalities such as CT, PET, and MRI.

9. Remote Operation: Finally, because robots are typically controlled by computers and/or remote electrical signals, the option exists to remotely operate the units over large distances through direct data links, or even over the internet (tele-robotics).

People have recognized many of these obvious advantages; therefore, we have seen a considerable increase in usage of robots in medical applications in recent times. As these advantages are general and apply to many medical procedures, the authors believe that it is just a matter of time before more robots are employed in automating a variety of procedures, ultimately increasing the quality while reducing the cost of medical care in the future [1-5].

Precision positioning systems have historically been a key part of successful medical societies. The need to make something requires the ability to move something with a very high level of accuracy. This has not changed in the Information Age, but instead has become even more important as global competition forces manufacturers to hold ever tighter specifications, with increased throughput and reduced costs. Automation is the equalizer that allows manufacturers in countries with high labor rates to compete globally with developing countries. The definition of a precision machine continues to evolve as technology advances. The components used to build machines become more precise, and so the machines themselves improve. As loosely defined here, precision machines are those that repeatable and reliably position to within a tolerance zone smaller than is possible in commonly available machines. Designing machines to best use the available components and manufacturing techniques requires specialized skills beyond those of general machine designers. It is interesting to note that many of the fundamental rules for designing precision machines have not changed for hundreds of years. Multi-degrees of freedom (DOF)-actuators have important role to have precision positioning. Multi-degreeof-freedom (DOF) actuators are finding wide use in a number of Industries. Currently, a significant number of the existing robotic actuators that can realize multi-DOF motion are constructed using gear and linkages to connect several single-DOF motors in series and/or parallel. Not only do such actuators tend to be large in size and mass, but they also have a decreased positioning accuracy due to mechanical deformation, friction and backlash of the gears and linkages. A number of these systems also exhibit singularities in their workspaces, which makes it virtually impossible to obtain uniform, high-speed, and highprecision motion. To improve the precision positioning new control methodology used in this research [6-9].

The application of control algorithms separates mechatronics systems and mechatronic design from traditional electromechanical systems. Modern controllers do more than simply close a PID loop. They provide additional options on feedback filtering and feedforward control, allow for coordination between multiple axes, and should provide an 
array of tuning and data collection utilities. Commercially available controllers generally use some form of classical proportional-integral-derivative (PID) control as their primary control architecture. This PID controller is usually coupled with one or more second-order digital filters to allow the user to design notch and low-pass filters for loop-shaping requirements. Classical sliding mode control is robust to model uncertainties and external disturbances. A sliding mode control method with a switching control law guarantees asymptotic stability of the system, but the addition of the switching control law introduces chattering into the system. One way of attenuating chattering is to insert a saturation function inside of a boundary layer around the sliding surface. Unfortunately, this addition disrupts Lyapunov stability of the closed-loop system. Classical sliding mode control method has difficulty in handling unstructured model uncertainties. One can overcome this problem by combining a sliding mode controller and fuzzy systems together. Fuzzy rules allow fuzzy systems to approximate arbitrary continuous functions. To approximate a time-varying nonlinear system, a fuzzy system requires a large amount of fuzzy rules. This large number of fuzzy rules will cause a high computation load. The addition of an adaptive law to a fuzzy sliding mode controller to online tune the parameters of the fuzzy rules in use will ensure a moderate computational load [10-15].

This research examines the low pass filter chattering free robust sliding mode controller. This type of active filter is used to reduce the high frequency oscillation (chattering) in this research and the role of fuzzy logic theory is to tuning the fuzzy logic coefficient. This paper is organized as follows; section 2, is served as a modeling and formulation of spherical motor, sliding mode controller and intro to fuzzy logic. Part 3, introduces and describes the control design to improve the system sensitivity in medical system. Section 4 presents the simulation results and discussion of this algorithm applied to medical actuator and the final section describe the conclusion.

\section{Theory}

\subsection{Spherical Motor}

Since its inception, the field of multi-DOF actuator dynamics has presented many issues in refining both theory and operations; one of the most challenging areas of study has been the problem of computational efficiency in the dynamics of mechanisms. Many efficient algorithms in dynamics have been developed to address this problem. The dynamics of multi-DOF actuators illustrate the relationship between force and motion. The generalized force for a multi-DOF actuator can be described as a second-order nonlinear differential equation. Several different methods are available to compute system dynamic equations. These methods include the Newton-Euler (N-E) methodology, the Lagrange-Euler (L-E) method, and Kane's methodology [16-20]. The Newton-Euler methodology is based on Newton's second law and several different researchers are signifying to develop this method. This equation can be described the behavior of a spherical motor joint-by-joint from first to final, called forward recursion and transfer the essential information from end to base frame, called backward recursive. The literature on Euler-Lagrange's is vast but a good starting point to learn about it is in[16-20]. Calculate the dynamic equation spherical motor using E-L method is easier because this equation is derivation of nonlinear coupled and quadratic differential equations. The Kane's method was introduced in 1961 by Professor Thomas Kane[18]. This method used to calculate the dynamic equation of motion without any differentiation between kinetic and potential energy functions. The dynamic equation of multi-DOF actuators is derived using the Lagrangian. The Lagrangian is derived by subtracting potential energy from kinetic energy. The dynamic equation of multi-DOF actuator governed by the following equation [20]: 


$$
\begin{aligned}
& H(q)\left[\begin{array}{c}
\ddot{\alpha} \\
\ddot{\beta} \\
\ddot{\gamma}
\end{array}\right]+B(q)\left[\begin{array}{c}
\dot{\alpha} \dot{\beta} \\
\dot{\alpha} \dot{\gamma} \\
\dot{\beta} \dot{\gamma}
\end{array}\right]+C(q)\left[\begin{array}{c}
\dot{\alpha}^{2} \\
\dot{\beta}^{2} \\
\dot{\gamma}^{2}
\end{array}\right]=\left[\begin{array}{c}
\tau_{x} \\
\tau_{y} \\
\tau_{z}
\end{array}\right] \\
& \text { - } \quad \tau \text { is actuation torque } \\
& \text { - } \quad H(q) \text { is a symmetric and positive define inertia matrix } \\
& \text { - } \quad \mathrm{B}(\mathrm{q}) \text { is the matrix of coriolios torques } \\
& \text { - } \quad \mathrm{C}(\mathrm{q}) \text { is the matrix of centrifugal torques. }
\end{aligned}
$$

The angular acceleration is found as to be:

$$
\left[\begin{array}{c}
\ddot{\alpha} \\
\ddot{\boldsymbol{\beta}} \\
\ddot{\gamma}
\end{array}\right]=H^{-1}(\boldsymbol{q}) \cdot\left\{\tau-\mathbf{B}(\boldsymbol{q})\left[\begin{array}{c}
\dot{\alpha} \dot{\beta} \\
\dot{\alpha} \dot{\gamma} \\
\dot{\boldsymbol{\beta}} \dot{\gamma}
\end{array}\right]-C(q)\left[\begin{array}{c}
\dot{\alpha}^{2} \\
\dot{\boldsymbol{\beta}}^{2} \\
\dot{\gamma}^{2}
\end{array}\right]\right\}
$$

\subsection{Sliding Mode Controller}

Sliding mode control theory for control joint of robot manipulator was first proposed in 1978 by Young to solve the set point problem $\left(\dot{\boldsymbol{q}}_{\boldsymbol{d}}=\mathbf{0}\right)$ by discontinuous method in the following form;

$$
\tau_{(q, t)}= \begin{cases}\tau_{i}^{+}(q, t) & \text { if } S_{i}>0 \\ \tau_{i}^{-}(q, t) & \text { if } S_{i}<0\end{cases}
$$

where $\boldsymbol{S}_{\boldsymbol{i}}$ is sliding surface (switching surface), $\boldsymbol{i}=\mathbf{1}, \mathbf{2}, \ldots \ldots, \boldsymbol{n}$ for $n$-DOF joint, $\boldsymbol{\tau}_{\boldsymbol{i}}(\boldsymbol{q}, \boldsymbol{t})$ is the $\boldsymbol{i}^{\boldsymbol{t h}}$ torque of joint. Sliding mode controller is divided into two main sub controllers:

- $\quad$ Corrective $\operatorname{control}\left(\boldsymbol{U}_{\boldsymbol{c}}\right)$

- $\quad$ Equivalent controller $\left(\boldsymbol{U}_{\boldsymbol{e q}}\right)$.

Discontinues controller causes an acceptable tracking performance at the expense of very fast switching. Conversely in this theory good trajectory following is based on fast switching, fast switching is caused to have system instability and chattering phenomenon. Fine tuning the sliding surface slope is based on nonlinear equivalent part [18]. However, this controller is used in many applications but, pure sliding mode controller has two most important challenges: chattering phenomenon and nonlinear equivalent dynamic formulation in uncertain parameters[19].

Chattering phenomenon can causes some problems such as saturation and heats the mechanical parts of joints or drivers. Design a robust controller for multi-DOF-joints is essential because these joints have highly nonlinear dynamic parameters. Consider a nonlinear single input dynamic system is defined by:

$x^{(n)}=f(\vec{x})+b(\vec{x}) u$

Where $\mathrm{u}$ is the vector of control input, $\boldsymbol{x}^{(\boldsymbol{n})}$ is the $\boldsymbol{n}^{\text {th }}$ derivation of $\boldsymbol{x}, \boldsymbol{x}=$ $\left[\boldsymbol{x}, \dot{\boldsymbol{x}}, \ddot{\boldsymbol{x}}, \ldots, \boldsymbol{x}^{(\boldsymbol{n}-1)}\right]^{\boldsymbol{T}}$ is the state vector, $\boldsymbol{f}(\boldsymbol{x})$ is unknown or uncertainty, and $\boldsymbol{b}(\boldsymbol{x})$ is of known sign function. The main goal to design this controller is train to the desired state; $\boldsymbol{x}_{\boldsymbol{d}}=\left[\boldsymbol{x}_{\boldsymbol{d}}, \dot{\boldsymbol{x}}_{\boldsymbol{d}}, \ddot{\boldsymbol{x}}_{\boldsymbol{d}}, \ldots, \boldsymbol{x}_{\boldsymbol{d}}^{(\boldsymbol{n}-\mathbf{1})}\right]^{\boldsymbol{T}}$, and trucking error vector is defined by [14]:

$\widetilde{x}=x-x_{d}=\left[\widetilde{x}, \ldots, \widetilde{x}^{(n-1)}\right]^{T}$

A time-varying sliding surface $\boldsymbol{s}(\boldsymbol{x}, \boldsymbol{t})$ in the state space $\boldsymbol{R}^{\boldsymbol{n}}$ is given by: 
$s(x, t)=\left(\frac{d}{d t}+\lambda\right)^{n-1} \widetilde{x}=0$

where $\lambda$ is the positive constant. To further penalize tracking error, integral part can be used in sliding surface part as follows:

$s(x, t)=\left(\frac{d}{d t}+\lambda\right)^{n-1}\left(\int_{0}^{t} \widetilde{x} d t\right)=0$

The main target in this methodology is kept the sliding surface slope $\boldsymbol{s}(\boldsymbol{x}, \boldsymbol{t})$ near to the zero. Therefore, one of the common strategies is to find input $\boldsymbol{U}$ outside of $\boldsymbol{s}(\boldsymbol{x}, \boldsymbol{t})$.

$\frac{1}{2} \frac{d}{d t} s^{2}(x, t) \leq-\zeta|s(x, t)|$

where $\zeta$ is positive constant.

If $\mathbf{S}(\mathbf{0})>\mathbf{0} \rightarrow \frac{\mathrm{d}}{\mathrm{dt}} \mathbf{S}(\mathbf{t}) \leq-\zeta$

To eliminate the derivative term, it is used an integral term from $t=0$ to $t=t_{\text {reach }}$

$\int_{t=0}^{t=t_{\text {reach }}} \frac{d}{d t} S(t) \leq-\int_{t=0}^{t=t_{\text {reach }}} \eta \rightarrow S\left(t_{\text {reach }}\right)-S(0) \leq-\zeta\left(t_{\text {reach }}-0\right)$

Where $t_{\text {reach }}$ is the time that trajectories reach to the sliding surface so, suppose $\mathrm{S}$ $\left(t_{\text {reach }}=0\right)$ defined as

$0-S(0) \leq-\eta\left(t_{\text {reach }}\right) \rightarrow t_{\text {reach }} \leq \frac{S(0)}{\zeta}$

And

if $\boldsymbol{S}(\mathbf{0})<0 \rightarrow 0-S(\mathbf{0}) \leq-\eta\left(\boldsymbol{t}_{\text {reach }}\right) \rightarrow \boldsymbol{S}(\mathbf{0}) \leq-\zeta\left(\boldsymbol{t}_{\text {reach }}\right) \rightarrow \boldsymbol{t}_{\text {reach }} \leq \frac{|\boldsymbol{S}(\mathbf{0})|}{\eta}$

Equation (12) guarantees time to reach the sliding surface is smaller than $\frac{|\boldsymbol{S}(\mathbf{0})|}{\zeta}$ since the trajectories are outside of $S(t)$.

if $S_{t_{\text {reach }}}=S(0) \rightarrow \operatorname{error}\left(x-x_{d}\right)=0$

Suppose $\mathrm{S}$ is defined as

$$
s(x, t)=\left(\frac{d}{d t}+\lambda\right)^{\mathbf{n}} \widetilde{x}=\left(\dot{\mathbf{x}}-\dot{\mathbf{x}}_{\mathbf{d}}\right)+\lambda\left(\mathbf{x}-\mathbf{x}_{\mathbf{d}}\right)
$$

The derivation of S, namely, $\dot{S}$ can be calculated as the following;

$\dot{S}=\left(\ddot{\mathbf{x}}-\ddot{\mathbf{x}}_{\mathbf{d}}\right)+\lambda\left(\dot{\mathbf{x}}-\dot{\mathbf{x}}_{\mathbf{d}}\right)$

suppose the second order system is defined as; 
$\ddot{x}=f+u \rightarrow \dot{S}=f+U-\ddot{x}_{d}+\lambda\left(\dot{\mathbf{x}}-\dot{\mathbf{x}}_{\mathrm{d}}\right)$

Where $\boldsymbol{f}$ is the dynamic uncertain, and also since $S=0$ and $\dot{S}=0$, to have the best approximation, $\widehat{\boldsymbol{U}}$ is defined as

$\widehat{\boldsymbol{U}}=-\widehat{\boldsymbol{f}}+\ddot{\boldsymbol{x}}_{\boldsymbol{d}}-\lambda\left(\dot{\mathbf{x}}-\dot{\mathbf{x}}_{\mathbf{d}}\right)$

A simple solution to get the sliding condition when the dynamic parameters have uncertainty is the switching control law:

$U_{d i s}=\widehat{U}-K(\vec{x}, t) \cdot \operatorname{sgn}(s)$

where the switching function $\mathbf{s g n}(\mathbf{S})$ is defined as

$\operatorname{sgn}(s)= \begin{cases}1 & s>0 \\ -1 & s<0 \\ 0 & s=0\end{cases}$

and the $\boldsymbol{K}(\overrightarrow{\boldsymbol{x}}, \boldsymbol{t})$ is the positive constant. Suppose by (7) the following equation can be written as,

$\frac{1}{2} \frac{d}{d t} s^{2}(x, t)=\dot{S} \cdot S=[f-\widehat{f}-K \operatorname{sgn}(s)] \cdot S=(f-\hat{f}) \cdot S-K|S|$

and if the equation (11) instead of (10) the sliding surface can be calculated as

$s(x, t)=\left(\frac{d}{d t}+\lambda\right)^{2}\left(\int_{0}^{t} \tilde{x} d t\right)=\left(\dot{x}-\dot{x}_{d}\right)+2 \lambda\left(\dot{x}-\dot{x}_{d}\right)-\lambda^{2}\left(x-x_{d}\right)$

in this method the approximation of $\boldsymbol{U}$ is computed as

$\widehat{U}=-\widehat{f}+\ddot{x}_{d}-2 \lambda\left(\dot{x}-\dot{x}_{d}\right)+\lambda^{2}\left(x-x_{d}\right)$

Based on above discussion, the sliding mode control law for multi-DOF-joints is written as:

$\boldsymbol{U}=\boldsymbol{U}_{e q}+\boldsymbol{U}_{\boldsymbol{c}}$

where, the model-based component $\boldsymbol{U}_{\boldsymbol{e q}}$ is the nominal dynamics of systems and calculated as follows:

$U_{e q}=\left[H^{-1}(q)\left(\mathbf{B}(q)\left[\begin{array}{c}\dot{\alpha} \dot{\beta} \\ \dot{\alpha} \dot{\gamma} \\ \dot{\beta} \dot{\gamma}\end{array}\right]+C(q)\left[\begin{array}{c}\dot{\alpha}^{2} \\ \dot{\beta}^{2} \\ \dot{\gamma}^{2}\end{array}\right]\right)+\dot{S}\right] H(q)$

and $\boldsymbol{U}_{\boldsymbol{c}}$ is computed as; 
$U_{c}=K \cdot \operatorname{sgn}(S)$

By (24) and (25) the sliding mode control of multi-DOF-joint is calculated as;

$$
\left[\begin{array}{c}
\widehat{\tau_{\alpha}} \\
\widehat{\tau_{\beta}} \\
\widehat{\boldsymbol{\tau}_{\gamma}}
\end{array}\right]=\left[H^{-1}(\boldsymbol{q})\left(\mathbf{B}(\boldsymbol{q})\left[\begin{array}{c}
\dot{\alpha} \dot{\beta} \\
\dot{\alpha} \dot{\gamma} \\
\dot{\beta} \dot{\gamma}
\end{array}\right]+C(q)\left[\begin{array}{c}
\dot{\alpha}^{2} \\
\dot{\beta}^{2} \\
\dot{\gamma}^{2}
\end{array}\right]\right)+\dot{S}\right] H(q)+K \cdot \operatorname{sgn}(S)
$$

Proof of Stability: The lyapunov formulation can be written as follows,

$$
V=\frac{1}{2} S^{T} . H . S
$$

the derivation of $V$ can be determined as,

$$
\dot{V}=\frac{1}{2} S^{T} \cdot \dot{H} \cdot S+S^{T} H \dot{S}
$$

the dynamic equation of multi-DOF actuator can be written based on the sliding surface as

$H \dot{S}=-V S+H \dot{S}+B+C$

it is assumed that

$S^{T}(\dot{H}-2 B+C) S=0$

by substituting (29) in (30)

$\dot{V}=\frac{1}{2} S^{T} \dot{H} S-S^{T} B+C S+S^{T}(H \dot{S}+B+C S)=S^{T}(H \dot{S}+B+C S)$

suppose the control input is written as follows

$\widehat{U}=\widehat{U_{\text {Nonlinear }}}+\widehat{U_{c}}=\left[\widehat{H^{-1}}(B+C)+\dot{S}\right] \widehat{H}+K \cdot \operatorname{sgn}(S)+B+C S$

by replacing the equation (32) in (25)

$\dot{V}=S^{T}\left(H \dot{S}+B+C-\widehat{H} \dot{S}-\widehat{B+C S}-K \operatorname{sgn}(S)=S^{T}(\widetilde{H} \dot{S}+\widetilde{B+C S}-K \operatorname{sgn}(S))\right.$

and

$|\widetilde{\boldsymbol{H}} \dot{\boldsymbol{S}}+\widetilde{\boldsymbol{B}+\boldsymbol{C} S}| \leq|\widetilde{\boldsymbol{H}} \dot{S}|+|\widetilde{B+C S}|$

the Lemma equation in multi-DOF actuator can be written as follows

$K_{u}=[|\widetilde{H} \dot{S}|+|B+C S|+\eta]_{i}, i=1,2,3,4, \ldots$

and finally;

$\dot{V} \leq-\sum_{i=1}^{n} \eta_{i}\left|S_{i}\right|$ 


\subsection{Fuzzy Logic Theory}

Supposed that $U$ is the universe of discourse and $x$ is the element of $U$, therefore, a crisp set can be defined as a set which consists of different elements $(x)$ will all or no membership in a set. A fuzzy set is a set that each element has a membership grade, therefore it can be written by the following definition;

$A=\left\{x, \mu_{A}(x) \mid x \in X\right\} ; A \in U$

Where an element of universe of discourse is $x, \mu_{A}$ is the membership function (MF) of fuzzy set. The membership function $\left(\mu_{A}(x)\right)$ of fuzzy set $A$ must have a value between zero and one. If the membership function $\mu_{A}(x)$ value equal to zero or one, this set change to a crisp set but if it has a value between zero and one, it is a fuzzy set. Defining membership function for fuzzy sets has divided into two main groups; namely; numerical and functional method, which in numerical method each number has different degrees of membership function and functional method used standard functions in fuzzy sets. The membership function which is often used in practical applications includes triangular form, trapezoidal form, bell-shaped form, and Gaussian form. A Trapezoidal membership function of fuzzy set is defined by the following equation

$\boldsymbol{\mu}_{\boldsymbol{F}(\boldsymbol{x})}=\left\{\begin{array}{cl}\mathbf{0}, \boldsymbol{a}, & x<a \\ \frac{\boldsymbol{b}-\boldsymbol{a}}{\boldsymbol{d}-\boldsymbol{x}}, & a \leq x<b \\ \frac{\boldsymbol{d}-\boldsymbol{c}}{\mathbf{0}}, & c \leq x<d \\ \mathbf{0}, & x>d\end{array}\right.$

A Triangular membership function of fuzzy set is defined by the following equation

$\boldsymbol{\mu}_{\boldsymbol{F}(\boldsymbol{x})}=\left\{\begin{array}{cl}\mathbf{0}, \boldsymbol{a} & x<a \\ \frac{\boldsymbol{b}-\boldsymbol{a}}{\boldsymbol{c}-\boldsymbol{x}}, & a \leq x<b \\ \frac{\boldsymbol{c}-\boldsymbol{b}}{\mathbf{0}}, & b \leq x \leq c \\ \mathbf{0}, & x>c\end{array}\right.$

A Gaussian membership function of fuzzy set is defined by

$\mu_{F(x)}=e^{\frac{-\left(x-c_{F}\right)^{2}}{W}}$

and a Bell-shaped membership function of fuzzy set is defined by

$\mu_{F(x)}=\frac{1}{1+\left(x-c_{F}\right)^{2}}$

The union of two fuzzy set $A$ and $B(S-$ norm $)$ is a new fuzzy set which the new membership function is given by

$\boldsymbol{S}(\boldsymbol{a}, \boldsymbol{b})=\boldsymbol{\mu}_{A \cup B(u)}=\max \left\{\boldsymbol{\mu}_{A(u)}, \boldsymbol{\mu}_{B(u)}\right\}, \quad \forall \boldsymbol{u} \in \boldsymbol{U}$

The intersection of two fuzzy set $A$ and $B(T-n o r m)$ is a new fuzzy set which the new membership function is given by

$$
\begin{aligned}
& T(a, b)= \mu_{A \cap B(u)}=\min \left\{\mu_{A(u)}, \mu_{B(u)}\right\}=\mu_{A(u) \cdot \mu_{B(u)}} \\
&=\max \left(0, \mu_{A(u)}+\mu_{B(u)}-1\right)= \begin{cases}\mu_{A(u)}, & \text { if } \mu_{B(u)}=1 \\
\mu_{B(u)}, & \text { if } \mu_{A(u)}=1 \\
0 \quad, \text { if } \mu_{B(u)}, \mu_{A(u)}<1\end{cases}
\end{aligned}
$$

In fuzzy set the min operation can resolve the statement $A A N D B$ and can be shown by $\min (A, B)$ operation. Using the same reason, the $A O R B$ operation can be replace by 
max operation in fuzzy set and at last the NOT $A$ operation can be replace by $1-A$ operation in fuzzy set. The algebraic product of two fuzzy set $A$ and $B$ is the multiplication of the membership functions which is given by the following equation

$$
\mu_{A . B(u)}=\mu_{A(u)} \cdot \mu_{B(u)}
$$

The algebraic Sum of two fuzzy sets $A$ and $B$ is given by the following equation

$$
\mu_{A \hat{+} B(u)}=\mu_{A(u)} \cdot \mu_{B(u)}-\mu_{A(u)} \cdot \mu_{B(u)}
$$

Linguistic variable can open a wide area to use of fuzzy logic theory in many applications (e.g., control and system identification). In a natural artificial language all numbers replaced by words or sentences.

If - then Rule statements are used to formulate the condition statements in fuzzy logic. A single fuzzy If - then rule can be written by

If $x$ is $A$ Then $y$ is $B$

where $A$ and $B$ are the Linguistic values that can be defined by fuzzy set, the If part of the part of " $x$ is $A$ " is called the antecedent part and the then - part of the part of " $y$ is $B$ " is called the Consequent or Conclusion part. The antecedent of a fuzzy if-then rule can have multiple parts, which the following rules shows the multiple antecedent rules:

\section{if $e$ is $N B$ and $\dot{e}$ is $M L$ then $T$ is $L L$}

where $e$ is error, $\dot{e}$ is change of error, $N B$ is Negative Big, $M L$ is Medium Left, $T$ is torque and $L L$ is Large Left.

If - then rules have three parts, namely, fuzzify inputs, apply fuzzy operator and apply implication method which in fuzzify inputs the fuzzy statements in the antecedent replaced by the degree of membership, apply fuzzy operator used when the antecedent has multiple parts and replaced by single number between 0 to 1 , this part is a degree of support for the fuzzy rule, and apply implication method used in consequent of fuzzy rule to replaced by the degree of membership.

The fuzzy inference engine offers a mechanism for transferring the rule base in fuzzy set which it is divided into two most important methods, namely, Mamdani method and Sugeno method. Mamdani method is one of the common fuzzy inference systems and he designed one of the first fuzzy controllers to control of system engine. Mamdani's fuzzy inference system is divided into four major steps: fuzzification, rule evaluation, aggregation of the rule outputs and defuzzification. Michio Sugeno use a singleton as a membership function of the rule consequent part. The following definition shows the Mamdani and Sugeno fuzzy rule base

$$
\begin{array}{rcccc}
\text { Mamdani } & F . R^{1}: \text { if } & x \text { is } A \text { and } y \text { is } B & \text { then } & z \text { is } C \\
\text { Sugeno } & F . R^{1}: \text { if } & x \text { is } A \text { and } y \text { is } B & \text { then } & f(x, y) \text { is } C
\end{array}
$$

When $x$ and $y$ have crisp values fuzzification calculates the membership degrees for antecedent part. Rule evaluation focuses on fuzzy operation $(A N D / O R)$ in the antecedent of the fuzzy rules. The aggregation is used to calculate the output fuzzy set and several methodologies can be used in fuzzy logic controller aggregation, namely, Max-Min aggregation, Sum-Min aggregation, Max-bounded product, Max-drastic product, Maxbounded sum, Max-algebraic sum and Min-max. Two most common methods that used in fuzzy logic controllers are Max-min aggregation and Sum-min aggregation. Max-min aggregation defined as below 
$\mu_{U}\left(x_{k}, y_{k}, U\right)=\mu_{\cup i=1}^{r} F R^{i}\left(x_{k}, y_{k}, U\right)=\max \left\{\min _{i=1}^{r}\left[\mu_{R}\left(x_{k}, y_{k}\right), \mu_{p_{m}}(U)\right]\right\}$

The Sum-min aggregation defined as below

$$
\mu_{U}\left(x_{k}, y_{k}, U\right)=\mu_{\cup_{i=1}^{r} F R^{i}}\left(x_{k}, y_{k}, U\right)=\sum \min _{i=1}^{r}\left[\mu_{R} p q\left(x_{k}, y_{k}\right), \mu_{p_{m}}(U)\right]
$$

where $r$ is the number of fuzzy rules activated by $x_{k}$ and $y_{k}$ and also $\mu_{\cup_{i=1}^{r} F R^{i}}\left(x_{k}, y_{k}, U\right)$ is a fuzzy interpretation of $i-t h$ rule. Defuzzification is the last step in the fuzzy inference system which it is used to transform fuzzy set to crisp set. Consequently defuzzification's input is the aggregate output and the defuzzification's output is a crisp number. Centre of gravity method (COG) and Centre of area method $(C O A)$ are two most common defuzzification methods, which COG method used the following equation to calculate the defuzzification

$\operatorname{COG}\left(x_{k}, y_{k}\right)=\frac{\sum_{i} U_{i} \sum_{j=1}^{r} \cdot \mu_{u}\left(x_{k}, y_{k}, U_{i}\right)}{\sum_{i} \sum_{j=1}^{r} \cdot \mu_{u}\left(x_{k}, y_{k}, U_{i}\right)}$

and $C O A$ method used the following equation to calculate the defuzzification

$\operatorname{COA}\left(x_{k}, y_{k}\right)=\frac{\sum_{i} U_{i} \cdot \mu_{u}\left(x_{k}, y_{k}, U_{i}\right)}{\sum_{i} \mu_{U} \cdot\left(x_{k}, y_{k}, U_{i}\right)}$

Where $\operatorname{COG}\left(x_{k}, y_{k}\right)$ and $\operatorname{COA}\left(x_{k}, y_{k}\right)$ illustrates the crisp value of defuzzification output, $U_{i} \in U$ is discrete element of an output of the fuzzy set, $\mu_{U}$. $\left(x_{k}, y_{k}, U_{i}\right)$ is the fuzzy set membership function, and $r$ is the number of fuzzy rules.

\section{Propopsed Methodology}

A low-pass filter is a filter that passes low-frequency signals and attenuates (reduces the amplitude of) signals with frequencies higher than the cutoff frequency. The actual amount of attenuation for each frequency varies depending on specific filter design. It is sometimes called a high-cut filter, or treble cut filter in audio applications. A low-pass filter is the opposite of a high-pass filter. A band-pass filter is a combination of a low-pass and a high-pass.

Low-pass filters exist in many different forms, including electronic circuits (such as a hiss filter used in audio), anti-aliasing filters for conditioning signals prior to analog-todigital conversion, digital filters for smoothing sets of data, acoustic barriers, blurring of images, and so on. The moving average operation used in fields such as finance is a particular kind of low-pass filter, and can be analyzed with the same signal processing techniques as are used for other low-pass filters. Low-pass filters provide a smoother form of a signal, removing the short-term fluctuations, and leaving the longer-term trend. An optical filter can correctly be called a low-pass filter, but conventionally is called a longpass filter (low frequency is long wavelength), to avoid confusion.

Chattering phenomenon is the main challenge in pure sliding mode controller with regards to the certain and partly uncertain situation. To reduce or eliminate the chattering in sliding mode controller, active low pass filter is used in this research. As their name implies, Active Filters contain active components such as operational amplifiers, transistors or FET's within their circuit design. They draw their power from an external power source and use it to boost or amplify the output signal. In this method the basic idea is filtered the discontinuous function by low pass active filter. 
$U=K(\vec{x}, t) \cdot \operatorname{Sgn}(S) \times \frac{1}{2 \pi \sqrt{C_{e q} \times R_{e q}}}$

Where $\boldsymbol{C}_{\boldsymbol{e q}} \boldsymbol{a n d} \boldsymbol{R}_{\boldsymbol{e q}}$ are different based on type of active filter. In this research second order active filter is used. Figure 1 shows the second order filter.

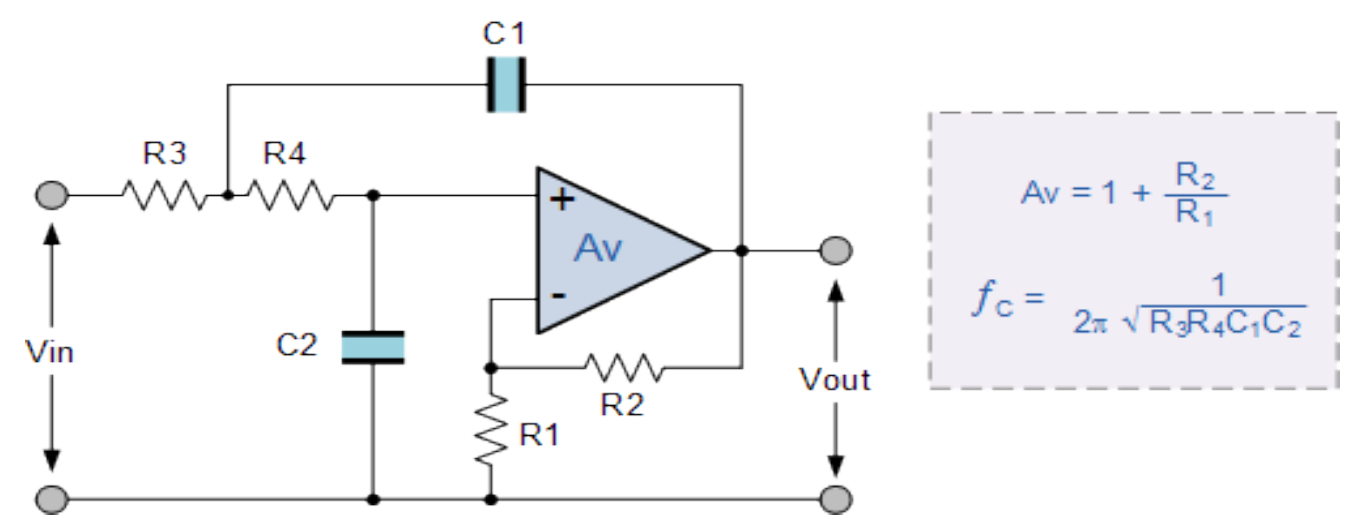

Figure 1. Second Order Active Filter

Regarding to above filter the rate of $\boldsymbol{R}_{\mathbf{1}} \boldsymbol{a n d} \boldsymbol{R}_{\mathbf{2}}$ used to calculate rate of filter gain and $\boldsymbol{C}_{1}, \boldsymbol{C}_{2}, \boldsymbol{R}_{\mathbf{3}} \boldsymbol{a n d} \boldsymbol{R}_{\mathbf{4}}$ used to design the low pass filter and cut-off frequency.

Based on above discussion, the control law for discontinuous part of multi degrees of freedom joints is written as

$\tau_{n e w-d i s}=K \cdot \operatorname{Sgn}(S) \times \frac{W}{2 \pi \sqrt{C_{e q} \times R_{e q}}}$

where

$\tau_{n e w-d i s}=\left[\begin{array}{l}\tau_{n e w-d i s 1} \\ \tau_{n e w-d i s 2} \\ \tau_{n e w-d i s 3}\end{array}\right], K=\left[\begin{array}{l}K_{1} \\ K_{2} \\ K_{3}\end{array}\right],(S)=\left[\begin{array}{l}S_{1} \\ S_{2} \\ S_{3}\end{array}\right]$ and $S=\lambda e+\dot{e}$

In some applications most, controllers are still classical linear or conventional nonlinear, but in uncertain condition design conventional controller with high performance response is very difficult and sometimes impossible. The first solution is applied artificial intelligence theory beside the robust linear and/or nonlinear algorithm in a limit variation. Conversely this solution is used in many applications it has some limitations such as limitation the nonlinear dynamic variation and design high performance intelligent controller. The second solution to improve the system performance is adaptive methodology. Adaptive (on-line) control is used in systems whose dynamic parameters are varying and need to be training on line. Based on above discussion, compute the best value of sliding surface slope coefficient, gain updating factor and filter gain have played important role to improve system's tracking performance especially when the system parameters are unknown or uncertain. In this research the high frequency oscillation reduces or eliminate based on first order filter, online tuning the filter's gain and sliding surface slope. This problem is solved by tuning the surface slope coefficient $(\boldsymbol{\lambda})$ and filter's gain $(\boldsymbol{W})$ by fuzzy logic theory. In this methodology, the system's performance is improved with respect to the classical sliding mode controller. To adjust the sliding surface slope and filter gain coefficients we define $\hat{\boldsymbol{f}}(\boldsymbol{x} \mid \boldsymbol{\lambda}, \boldsymbol{W})$ as the fuzzy based tuning. 


$$
\hat{f}(x \mid \lambda)=\lambda^{T} \zeta(x)
$$

If minimum error $\left(\boldsymbol{\lambda}^{*}, \boldsymbol{W}^{*}\right)$ is defined by;

$$
\begin{aligned}
& \lambda^{*}=\arg \min [(\operatorname{Sup} \mid \hat{f}(x \mid \lambda)-f(x))] \\
& W^{*}=\arg \min [(\operatorname{Sup} \mid \hat{f}(x \mid W)-f(x))]
\end{aligned}
$$

where $\lambda^{T}$ is adjusted by an adaption law and this law is designed to minimize the error's parameters of $\boldsymbol{\lambda}-\boldsymbol{\lambda}^{*}$ and $\boldsymbol{W}-\boldsymbol{W}^{*}$. Adaption law in proposed method is used to on-line tuning the sliding surface slope and filter's gain coefficients.

$$
\begin{gathered}
S=(\dot{e}+\lambda e) \\
S_{\text {new }}=((1-\alpha) \times \dot{e}+\alpha \times \lambda e) 1 \\
S_{\text {new }}=(\alpha \times \lambda e-\alpha \times \dot{e}+\dot{e}) \\
\tau_{\text {new-dis }}=K \cdot \operatorname{Sgn}\left(S_{\text {new }}\right) \times \frac{W_{\text {new }}}{2 \pi \sqrt{C_{e q} \times R_{e q}}} \\
W_{\text {new }}=(\alpha \times W)
\end{gathered}
$$

Where $\boldsymbol{\alpha}$ is adaptive fuzzy output.

\subsection{Design Fuzzy Adaptive Technique}

In this research fuzzy based adaptive technique has two inputs error and change of error $(\boldsymbol{e}, \dot{\boldsymbol{e}})$ and the output name's is gain updating factor $(\boldsymbol{\alpha})$. The linguistic variables for error change of error and gain updating factor are; Negative Big (NB), Negative Medium (NM), Negative Small (NS), Zero (Z), Positive Small (PS), Positive Medium (PM), Positive Big (PB), and it is quantized into thirteen levels represented by: $-1,-0.83,-0.66$, $0.5,-0.33,-0.16,0,0.16,0.33,0.5,0.66,0.83,1$. In this research triangular membership function is selected because it has linear equation with regard to has a high-quality response. The rule base for gain updating factor is defined based on the following detail;

F.R1: IF $e$ is NB and $\dot{e}$ is NB, THEN $\alpha$ is PS.

After design rule-table for gain updating factor, COG method is used to defuzzification.

\section{Results and Discussion}

To evaluate the designed controller, repetitive simulation tests were performed via numerical simulation. Figure 2 to Figure 4 show the simulation result when the sliding surface $=10$. 


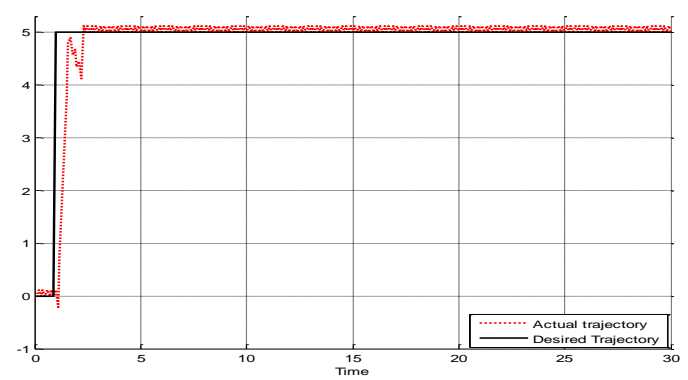

Figure 2. Trajectory Following in Classical SMC $(\lambda=10)$

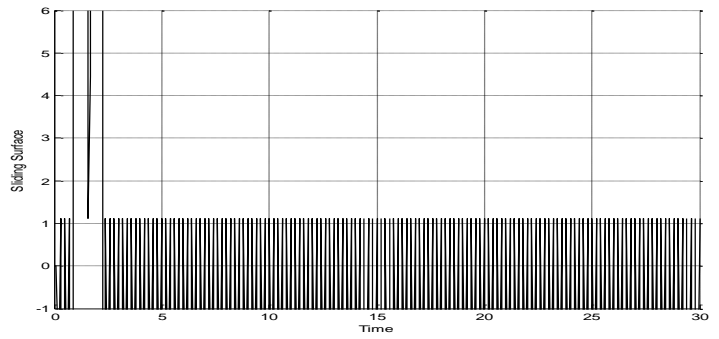

Figure 3. Sliding Surface Tracking $(\lambda=10)$

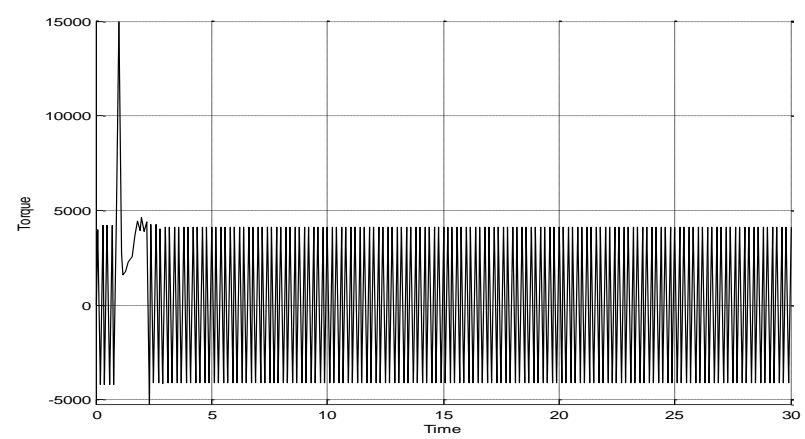

Figure 4. Torque Performance $(\lambda=10)$

Regarding to Figure 2 to 4, sliding mode controller has fluctuations in presence of certain and uncertain conditions. Sliding mode controller's performance is working based on sliding surface slope (coefficient). Figure 5 to Figure 7 show the results of the simulation with increase the slope of the sliding surface from 10 to 25 .

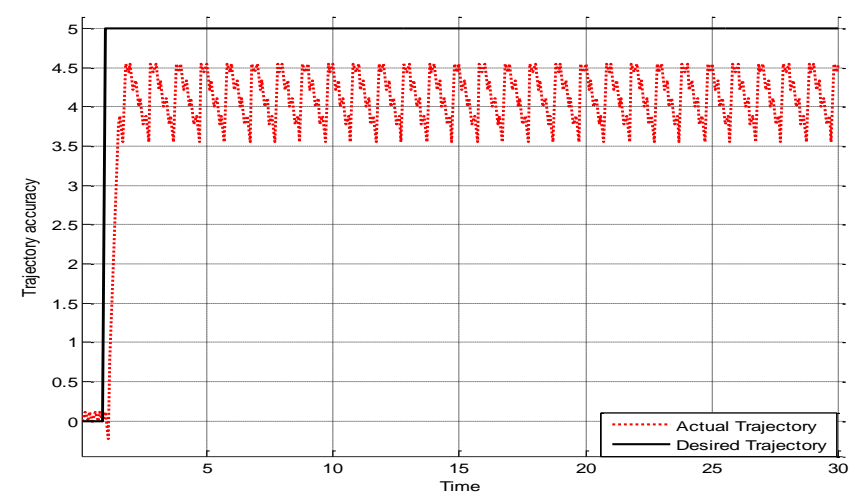

Figure 5. Trajectory Following in Classical SMC $(\lambda=25)$ 
According to Figure 2 and 5, it is clear that; sliding surface slope optimization is very important in sliding mode controller.

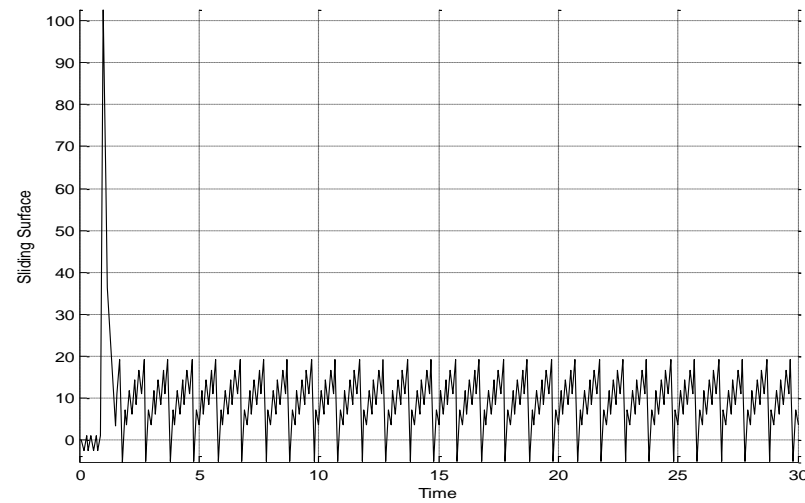

Figure 6. Sliding Surface Tracking $(\lambda=25)$

Regarding to Figure 3 and 6 (sliding surface tracking) with different sliding surface slope and based on stability definition in sliding mode controller, fluctuation in sliding surface tracking is depend on the sliding surface slope and optimize it play important role in stability.

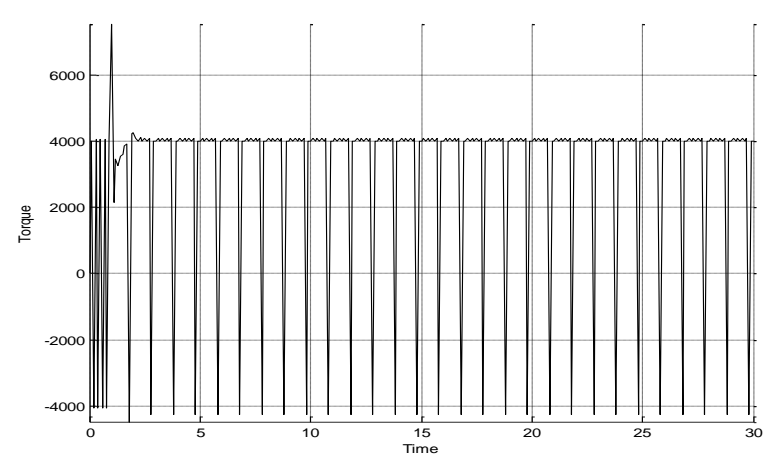

Figure 7. Torque Control $(\lambda=25)$

The oscillations in Figure 4 and Figure 7 show that different sliding surface slope has a different oscillation that appears in the torque control system.

Figure 8 to Figure 10 show the results of the simulation for the proposed method with optimization of sliding surface slope in certain and un-certain condition.

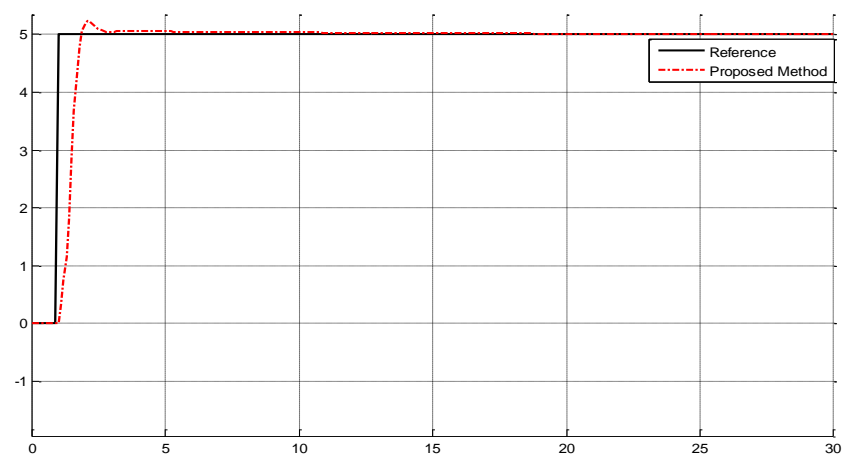

Figure 8. Trajectory Following in Proposed Method 


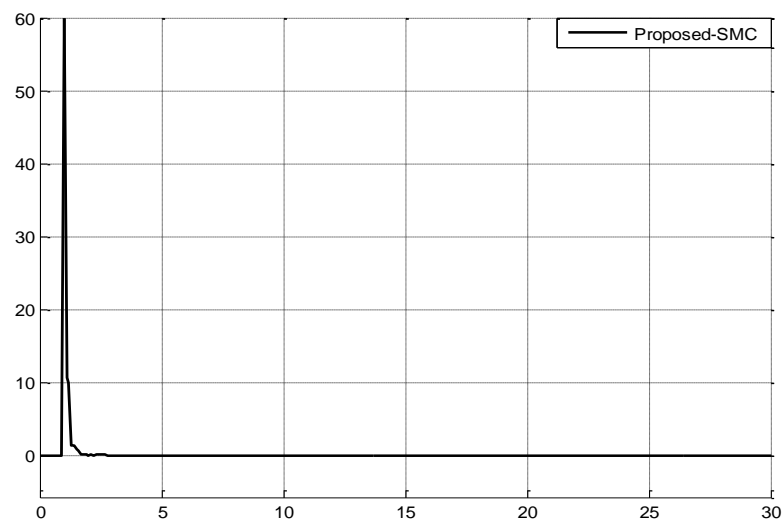

Figure 9. Sliding Surface Tracking

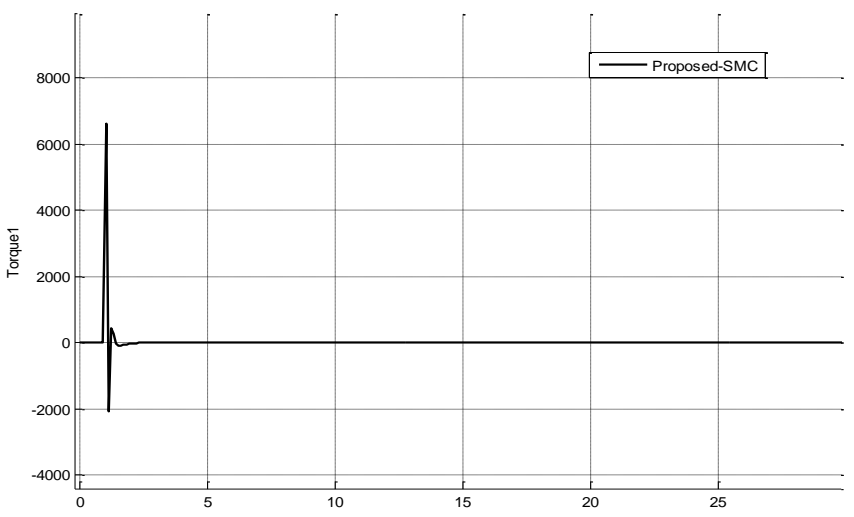

Figure 10. Torque Control Proposed Method

Regarding to Figure 7 to 10, on-line tuning the sliding surface slope and filter coefficient caused to eliminate the chattering beside to reduce the error. Based on medical industries sensitivity and vibration rejection are very important. This method succeeds in eliminating the chatter phenomenon as well improve the accuracy.

\section{Conclusion}

According to this paper, design a high performance, vibration rejection is introduce. Regarding to methodology, proposed sliding mode controller has two new parts: low pass filter to eliminate the high frequency oscillation which caused to vibration and intelligent tuning coefficient to on-line tuning the coefficient. Regarding to results and discussion, proposed sliding mode controller eliminate the chattering which caused to vibration. However low pass filter has the main role to reduce/eliminate the chattering but the filter coefficient and sliding surface slope optimization are also very important. Intelligent fuzzy logic methodology used to on-line tuning coefficients. Regarding to results, on-line tuning caused to improve the system performance in presence of uncertainty.

\section{Acknowledgment}

The authors would like to thank the anonymous reviewers for their careful reading of this paper and for their helpful comments. This work was supported by the Iranian Institute of Advance Science and Technology Program of Iran under grant no. 2014Persian Gulf-1A. 
Iranian center of Advance Science and Technology (IRAN SSP) is one of the independent research centers specializing in research and training across of Control and Automation, Electrical and Electronic Engineering, and Mechatronics \& Robotics in Iran. At IRAN SSP research center, we are united and energized by one mission to discover and develop innovative engineering methodology that solve the most important challenges in field of advance science and technology. The IRAN SSP Center is instead to fill a long standing void in applied engineering by linking the training a development function one side and policy research on the other. This center divided into two main units:

- $\quad$ Education unit

- $\quad$ Research and Development unit

\section{References}

[1] G. I. Vachtsevanos, K. Davey and K. M. Lee, "Development of a Novel Intelligent Robotic Manipulator," IEEE Control System Magazine, (1987), pp. 9-15.

[2] K. Davey, G. I. Vachtsevanos, and R. Powers, "An analysis of Fields and Torques in Spherical Induction Motors," IEEE Transactions on Magnetics, vol. no. 23, (1987), pp. 273-282.

[3] A. Foggia, E. Oliver and F. Chappuis, "New Three Degrees of Freedom Electromagnetic Actuator," Conference Record -lAS Annual Meeting, vol. 35, New York, (1988).

[4] K. M. Lee, G. Vachtsevanos and C. K. Kwan, "Development of a Spherical Wrist Stepper Motor," Proceedings of the 1988 IEEE lntemational Conference on Robotics and Automation, Philadelphia, PA, April 26-29.

[5] K. M. Lee and I. Pei, "Kinematic Analysis of a Three Degree-of-Freedom Spherical Wrist Actuator," The Fifth International Conference on Advanced Robotics, Italy, (1991).

[6] I. Wang, G. Jewel and D. Howe, "Modelling of a Novel Spherical Penn anent Magnet Actuator," Proceedings of IEEE International Conference on Robotics and Automation, Albuquerque, New Mexico, pp. 1190-1195, (1997).

[7] I. Wang, G. Jewel and D. Howe, "Analysis, Design and Control of a Novel Spherical Pennanent Magnet Actuator," lEE Proceedings on Electrical Power Applications., vol. 154, no. 1, (1998).

[8] G. S. Chirikjian and D. Stein, "Kinematic Design and Commutation of a Spherical Stepper Motor," IEEE IASME Transactions on Mechatronics, vol. 4, no. 4, Piscataway, New Jersey, pp. 342-353, Dec. 1999.

[9] K. Kahlen, and R. W. D. Doncker, "CW'l'ent Regulators for Multi-phase Penn anent Magnet Spherical Machines." Industry Applications Conference Record of the 2000 IEEE, vol. 3, (2000), pp. 2011-2016.

[10] K. M. Lee, I. Pei and U. Gilboa, "On the Development of a Spherical Wrist Actuator," Proceedings of the 16th NSF Conference on Manufacturing Systems Research, Tempe AZ, January 8-12, (1990).

[11] Z. Liu, H. Su and S. Pan, "A new adaptive sliding mode control of uncertain nonlinear systems", Asian Journal of Control, (2014), vol. 16, no. 1, 198-208.

[12] Y. Shang, "Consensus recovery from intentional attacks in directed nonlinear multi-agent systems", International Journal of Nonlinear Sciences and Numerical Simulation, (2013), vol. 14, no. 6, 355-361.

[13] F. Piltan, A. R. Salehi and N. B. Sulaiman, "Design Artificial Robust Control of Second Order System Based on Adaptive Fuzzy Gain Scheduling”, World Applied Science Journal (WASJ), vol. 13, no. 5, (2011), pp. 1085-1092. (ISI, Scopus, SJR=0.22, Q2).

[14] M. N. Kamarudin, A. R. Husain and M. N. Ahmad, "Control of uncertain nonlinear systems using mixed nonlinear damping function and back stepping techniques", 2012 IEEE International Conference on Control Systems, Computing and Engineering, Malaysia, (2012).

[15] N. F. Piltan, N. Sulaiman, S. Soltani, M. H. Marhaban and R. Ramli, "An Adaptive Sliding Surface Slope Adjustment in PD Sliding Mode Fuzzy Control For Robot Manipulator", International Journal of Control and Automation, vol. 4, no. 3, (2011), pp. 65-76, 2011, (Scopus, SJR=0.25., Q3)

[16] A. Siahbazi, A. Barzegar, M. Vosoogh, A. M. Mirshekaran and S. Soltani, "Design Modified Sliding Mode Controller with Parallel Fuzzy Inference System Compensator to Control of Spherical Motor", IJISA, vol.6, no.3, (2014), pp.12-25, DOI: 10.5815/ijisa.2014.03.02.

[17] M. Yaghoot, F. Piltan, M. Esmaeili, M. A. Tayebi and M. Piltan, "Design Intelligent Robust Modelbase Sliding Guidance Controller for Spherical Motor", IJMECS, vol.6, no.3, (2014), pp.61-72,.DOI: 10.5815/ijmecs.03.08

[18] S. Rahbar, F. Piltan, E. Pouladi, H. Davarpanah and S. Jowkar, "The Fuzzy (PI+D) 2 Sliding Mode Scheme to Motor Vibration Control", International Journal of U- and e- Service, Science and Technology, vol. 8, no. 1, (2015), pp. 371-388, http://dx.doi.org/10.14257/ijunesst.2015.8.1.33.

[19] F. Matin, F. Piltan, H. Cheraghi, N. Sobhani and M. Rahmani, "Design Intelligent PID like Fuzzy Sliding Mode Controller for Spherical Motor", IJIEEB, vol. 6, no. 2, (2014), pp. 53-63, DOI: 10.5815/ijieeb.2014.02.07. 
[20] S. Yauldegar, H. Ghiasi, M. H. Mazloom, A. Sahamijoo, M. R. Avazpour and F. Piltan, "Trajectory Tracking Control of Multi Degrees of Freedom Joints: Robust Fuzzy Logic-Based Sliding Mode Approach", International Journal of Control and Automation, vol. 7, no. 12, (2014), pp. 323-338, (Scopus, SJR=0.25, Q3).

\section{Authors}

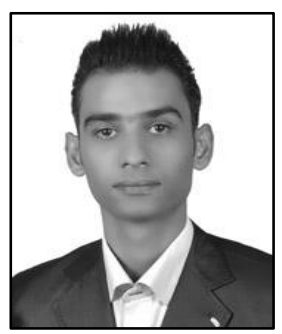

Mohammad Reza Avazpour, is currently a co researcher in the Intelligent System and Robotic Lab at Iranian Institute of Advance Science and Technology (IRAN SSP) of the program for design high precision and fast dynamic controller for multi-DOF- actuators.

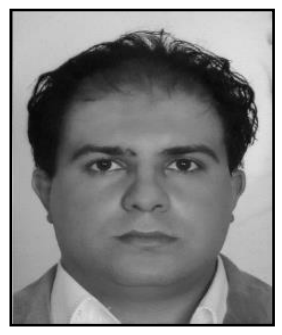

Farzin Piltan, was born on 1975, Shiraz, Iran. In 2004 he is jointed Institute of Advance Science and Technology, Research and Development Center, IRAN SSP. Now he is a dean of Intelligent Control and Robotics Lab. He is led of team (47 researchers) to design and build of nonlinear control of industrial robot manipulator for experimental research and education and published about 54 Papers in this field since 2010 to 2012, team supervisor and leader (9 researchers) to design and implement intelligent tuning the rate of fuel ratio in internal combustion engine for experimental research and education and published about 17 Journal papers since 2011 to 2013, team leader and advisor (34 researchers) of filtering the hand tremors in flexible surgical robot for experimental research and education and published about 31 journal papers in this field since 2012 to date, led of team (21 researchers) to design high precision and fast dynamic controller for multi-degrees of freedom actuator for experimental research and education and published about 7 journal papers in this field since 2013 to date, led of team (22 researchers) to research of full digital control for nonlinear systems (e.g., Industrial Robot Manipulator, IC Engine, Continuum Robot, and Spherical Motor) for experimental research and education and published about 4 journal papers in this field since 2010 to date and finally led of team (more than 130 researchers) to implementation of Project Based-Learning project at IRAN SSP research center for experimental research and education, and published more than 110 journal papers since 2010 to date. In addition to 7 textbooks, Farzin Piltan is the main author of more than 115 scientific papers in refereed journals. He is editorial review board member for 'international journal of control and automation (IJCA), Australia, ISSN: 2005-4297; 'International Journal of Intelligent System and Applications (IJISA)', Hong Kong, ISSN: 2074-9058; 'IAES international journal of robotics and automation, Malaysia, ISSN: 2089-4856; 'International Journal of Reconfigurable and Embedded Systems', Malaysia, ISSN: 20894864. His current research interests are nonlinear control, artificial control system and applied to FPGA, robotics and artificial nonlinear control and IC engine modeling and control. 